\title{
CineDocNet, TrDocNet: una comunidad virtual de usuarios de documentación audiovisual (cinematográfica y televisiva)
}

\author{
Por Alfonso López Yepes y José Ramón Pérez Agüera
}

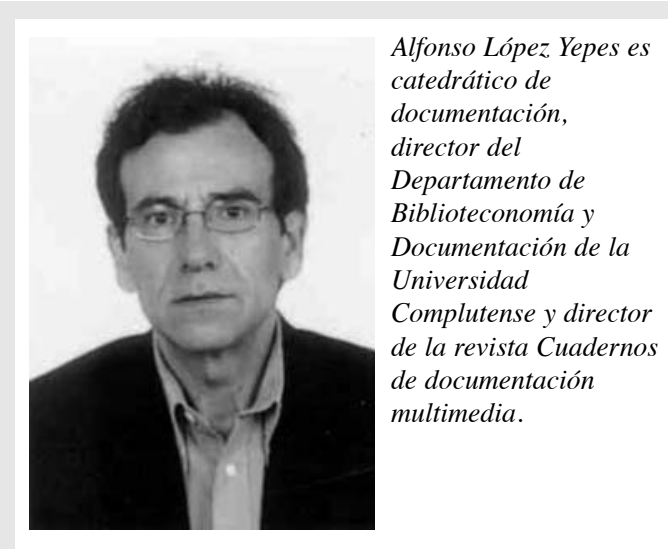

Resumen: La documentación informativa debe estar contemplada en todos sus ámbitos profesionales a través de una adecuada comunidad virtual de usuarios que integre docentes, investigadores, técnicos, críticos, documentalistas, profesionales en general, estudiantes, etc. Existe, en ciernes todavía, una comunidad virtual de usuarios de documentación que presenta, desde nuestro punto de vista, una concepción excesivamente amplia que debería contemplar de forma específica los diferentes ámbitos de la documentación. Este trabajo presenta una CVU de documentación audiovisual, ya en funcionamiento, que integra las vertientes cinematográfica y televisiva, caracterizada por sus contenidos multimedia, su interactividad y la integración de varias áreas de trabajo: documentos en línea, weblog, lista de distribución, foro, envío de aportaciones (moderadas) por parte de los usuarios y chat periódico. Para la producción y difusión de los contenidos multimedia se están utilizando sendos servidores de "streaming", el del centro de cálculo de la Univ. Com-

plutense de Madrid (UCM) y el de RedIris. La puesta en marcha de esta CVU se enmarca en un proyecto más amplio de establecimiento de una "Red española e iberoamericana de documentación multimedia", sugerida desde el Dpto. de Biblioteconomía y Documentación de la UCM.

Palabras clave: $C V U$, documentación informativa, documentación audiovisual, documentación cinematográfica, documentación televisiva, RedIris, CineDocNet, TvDocNet.

Title: CineDocNet, TrDocNet: a virtual community of audio-visual information management for cinema and television

\begin{abstract}
Media information management should be considered from all professional perspectives through virtual communities of users (VCU) made up of researchers, technical experts, critics, information and media professionals, students, etc. Although a new virtual community of information management has already been set up, in the opinion of the authors it is too broad to allow for sufficient attention to different areas of specialisation. The present article presents the case of an existing virtual community of users of audio-visual information, covering both cinematography and TV. This VCU is characterised by multimedia contents, interactivity, and the integration of distinctive work areas, such as a documentation service, web log, mail list, forum, users' contributions, and periodic chats. Two streaming servers are being used to produce and broadcast multimedia content: one located at RedIRIS [the Spanish National Research Network] and the other at the Computer Centre of the Univ. Complutense de Madrid (UCM). The VCU has its origins in a broader framework, proposed by the Library and Information Science Department of the UCM, whose aim is to establish a Spanish and Iberoamerican multimedia information management network.
\end{abstract}

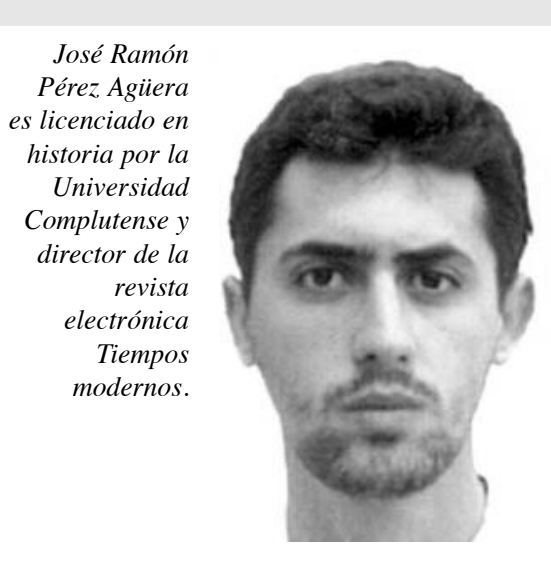

Keywords: $\boldsymbol{V C U}$, Virtual community of media information management, Audio-visual information management, Television information management, RedIris, CineDocNet, TvDocNet.

López Yepes, Alfonso; Pérez Agüera, José Ramón. “CineDocNet, TvDocNet: una comunidad virtual de usuarios de documentación audiovisual (cinematográfica y televisiva)”. En: El profesional de la información, 2002, septiembre-octubre, v. 11, n. 5, pp. 339-348.

Artículo recibido el 24-06-02

Aceptación definitiva: 23-07-02 


\section{Introducción}

Desde la creación de Well' en 1985, el crecimiento de las comunidades virtuales de usuarios ha sido impresionante. Millones de personas se han conectado mediante este sistema organizando una sociedad paralela dentro de los límites de internet. Hoy, 17 años después, el concepto de comunidad virtual de usuarios (CVU) ha tomado nuevos sentidos y se ha diversificado adaptándose a las necesidades y objetivos de los usuarios.

\section{«Se encuentra en funciona- miento una CVU de documenta- ción audiovisual que integra dos vertientes temáticas, cine- matográfica y televisiva»}

La dinámica de estas CVUs ha seguido las mismas líneas de desarrollo y evolución que los archiconocidos grupos sociales, reconocidos en sociología como unas de las unidades fundamentales para el estudio y comprensión de nuestra sociedad. No es momento ni lugar de definir las características generales del grupo social, y menos a tenor de las continuas discusiones que ha provocado entre los sociólogos su definición y tratamiento. Sin embargo, nuestro planteamiento se basa en la dinámica general que establecen los sociólogos a la hora de estudiarlos. Desde nuestro punto de vista partimos de las características generales del grupo social para entender la CVU, y posteriormente aplicar un grado más de especificidad centrándonos en la dinámica completa de lo que nosotros concebimos como una CVU de documentación informativa, en consonancia con los avances tecnológicos que hacen posible el funcionamiento de este ámbito temático.

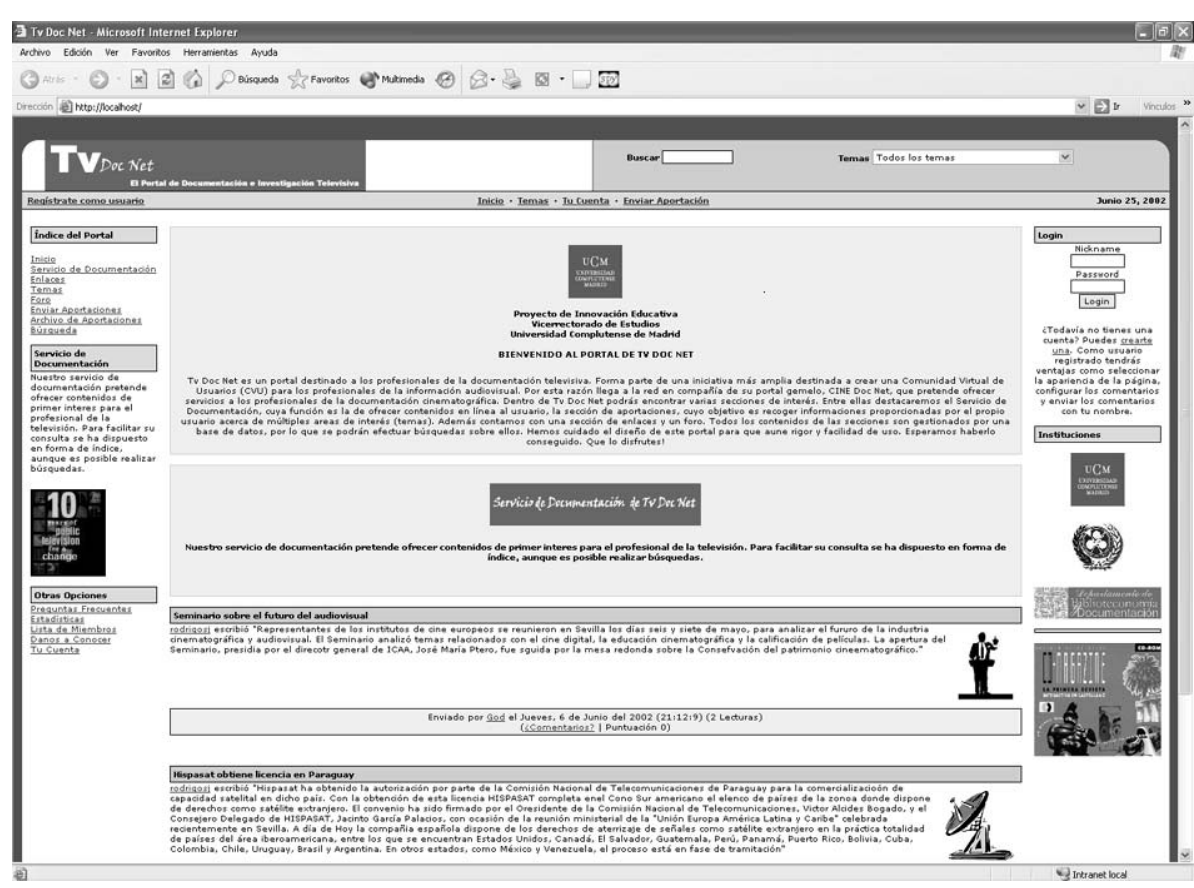

\section{La necesidad de una CVU de documentación informativa}

Como apunta muy acertadamente Manuel Castells $^{2}$, en el nuevo modo de desarrollo informacional en el que nos hallamos insertos la principal fuente de productividad estriba en la tecnología de la generación del conocimiento, el procesamiento de la información y la comunicación de símbolos. Para este autor es específico del modo de desarrollo informacional la acción del conocimiento sobre sí mismo como principal fuente de productividad. El procesamiento de la información se centra en el perfeccionamiento de la tecnología de ese procesamiento como fuente de productividad, en un círculo realimentado de interacción de las fuentes del conocimiento y el procesamiento de la información. Si reducimos la escala sociológica que utiliza Castells y nos aproximamos a la formación de grupos sociales concretos observamos que las afirmaciones del sociólogo catalán nos son de enorme utilidad en un entorno más reducido.

\section{«CineDocNet y TvDocNet se ca- racterizan por sus contenidos multimedia, su interactividad y la integración de varias áreas de trabajo»}

En el caso de los profesionales de la documentación informativa, siguiendo las líneas generales anteriormente expuestas, destaca su necesidad de adecuación a los nuevos entornos económicos y sociales de la nueva sociedad informacional lo cual hace, si cabe, aún más necesaria la creación de herramientas basadas en los avances tecnológicos que le acerquen a la realidad social de la que no sólo forma parte, sino de la que incluso es uno de sus principales protagonistas. Una CVU no es ni más ni menos que la acomodación de los avances tecnológicos al nuevo papel de la documentación informativa dentro de una nueva sociedad en la que la información es el nuevo capital y los documentalistas, de alguna forma, los nuevos banqueros.

Pese a que estas primeras líneas puedan resultar demasiado teóricas, no nos centraremos aquí en aspectos abstractos de las CVUs, sino más bien en la vertiente más práctica y concreta. Para ello haremos referencia a un proyecto que ya está en marcha y en cuyo desarrollo se despliegan las bases 
teóricas aquí esbozadas. Así pues, trataremos de definir los pasos fundamentales para la formación de una CVU de documentación audiovisual, como parte indispensable de la CVU de documentación informativa cuyo proceso de implantación no supone otra cosa que una definición de la escalabilidad de este proyecto. Nos referiremos por tanto a CineDocNet y TvDocNet, áreas en las que en la actualidad hemos dividido la CVU de documentación audiovisual que estamos preparando y de la cual constituyen la primera fase ya en desarrollo avanzado.

http://cinedocnet.rediris.es

http://tvdocnet.rediris.es

http://documentacionaudiovisual.rediris.es

\section{Características generales de las CVUs}

A la hora de prediseñar una CVU, entendida como herramienta colectiva, hemos de tener en cuenta una serie de características primordiales para garantizar su permanencia. En primer lugar se ha de fijar su grado de autonomía, lo que se define por la capacidad de ser entendida en sí misma sin dependencia de grupos o instituciones concretos que condicionen su desarrollo natural. Para ello hemos planteado esta iniciativa como un proyecto de todos que, si bien lo han puesto en marcha determinados organismos, no debe perder en ningún momento su total autonomía. La vía más eficaz para mantener esta libertad no es otra que la integración de las diferentes empresas, universidades, instituciones y profesionales autónomos en la CVU en grado de absoluta igualdad y con independencia de sus lugares de origen, para eneque así su desarrollo esté garantizado en todo momento sin que afecten las variaciones a las que se vean sometidos cada uno de sus miembros por separado.

\section{Streaming}

Es un sistema de transmisión de grandes ficheros (usualmente de audio o vídeo) a través de las redes, de manera que se produce un flujo constante entre el emisor y el receptor. La limitación de los actuales anchos de banda de la mayoría de usuarios para recibir vídeo se solventa con mejores algoritmos de compresión, así como, por ahora, rebajando un poco la calidad.
La homogeneidad dentro del grupo es otra de las características esenciales para su funcionamiento. La aplicación de unos principios de uniformidad garantiza el mantenimiento del interés por parte de cada uno de sus integrantes, ya que una excesiva heterogeneidad lleva a una dispersión de objetivos e intereses de cada uno de los miembros provocando su salida como usuarios activos. El problema que nos encontramos al fijar el grado de equilibrio es precisamente el de la escalabilidad, al tener que introducir subcomunidades dentro de la CVU que respondan a los intereses de los miembros en equilibrio entre la igualdad y la especialización. La respuesta concreta a este problema dentro de una CVU de documentación informativa ha sido la división entre cada uno de los subgrupos que la forman y, una vez ahí, la reproducción de las características de todos esos colectivos.

\section{«Para la producción y difusión de los contenidos multimedia se están utilizando sendos ser- vidores de streaming, el del centro de cálculo de la UCM y el de RedIris"}

Otro aspecto básico es la flexibilidad, que debe permitir variaciones al conjunto de usuarios sin llegar al grado de ruptura que provocaría su disolución. Para esto hace falta una voluntad de adaptación a las necesidades de los usuarios por parte de los impulsores de la CVU, ya que si no es así permanece rígidamente asentada en sus bases primigenias, quedando reducida a un grupo pequeño de miembros y perderá el interés para la mayoría, que la abandonará. La CVU no sería capaz de aprovechar las sinergias de los propios profe- 
sionales. Por esta razón es fundamental un sentido permanente de actualidad que haga de la CVU un centro de información que aporte a cada uno de los usuarios un flujo constante de nuevos conocimientos. Esto supone que cada uno de los miembros no sólo acuda a ella en busca de materiales concretos sino que la visite cotidianamente como una actividad necesaria para el reciclaje y la puesta al día de su profesión.

El grado de participación debe ser alto para que la CVU perviva. En muchas de las que existen en la actualidad, sobre todo en aquellas de formación más artificial, un reducido grado de par-

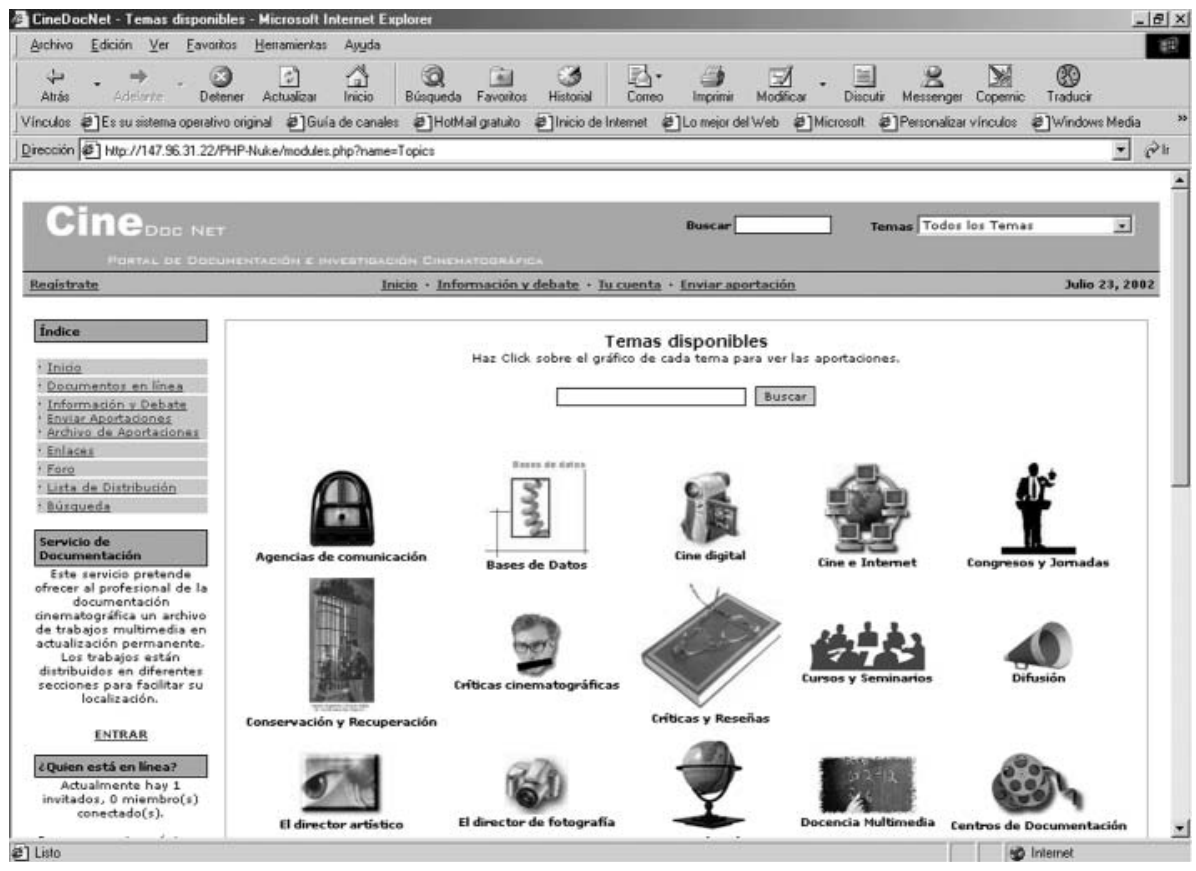
ticipación hace que tengan una existencia más bien callada y fracasen en su papel de gestoras y productoras de información, convirtiéndose en herramientas mantenidas por unos pocos y utilizadas de forma irregular por una mayoría. Para que los integrantes se mantengan interesados debe existir un grado de intimidad dentro de la CVU que evite la producción de ruido, lo que provocaría una pérdida de interés por su parte.

En el caso concreto de las CVUs citadas, el perfil de estos usuarios sería el de responsables y personal de servicios de documentación de televisión, profesionales de filmotecas y centros de documentación cinematográfica, estudiantes, docentes e investigadores, profesionales cinematográficos con necesidad de informarse y documentarse y, en general, cualquier profesional interesado en estos ámbitos.

En consecuencia, la participación de miembros ha de ser regulada por la propia dinámica de la CVU. Así, por ej., no debe ser utilizada CineDocNet por aficionados no profesionales, que la concibieran como otro portal sobre cine, entendida más como un elemento de ocio que de uso profesional. En los primeros momentos de su formación el papel de los administradores es vital para mantener un filtrado de ruido que permita el lanzamiento de una dinámica de profesionales que se mantendrá por sí sola cuando se haya consolidado. Una vez contemplado esto, está claro que la pervivencia de una CVU de documentación informativa está íntimamente ligada a la formación de subgrupos o CVUs subordinadas a la principal que permitan articular las características anteriormente mencionadas.

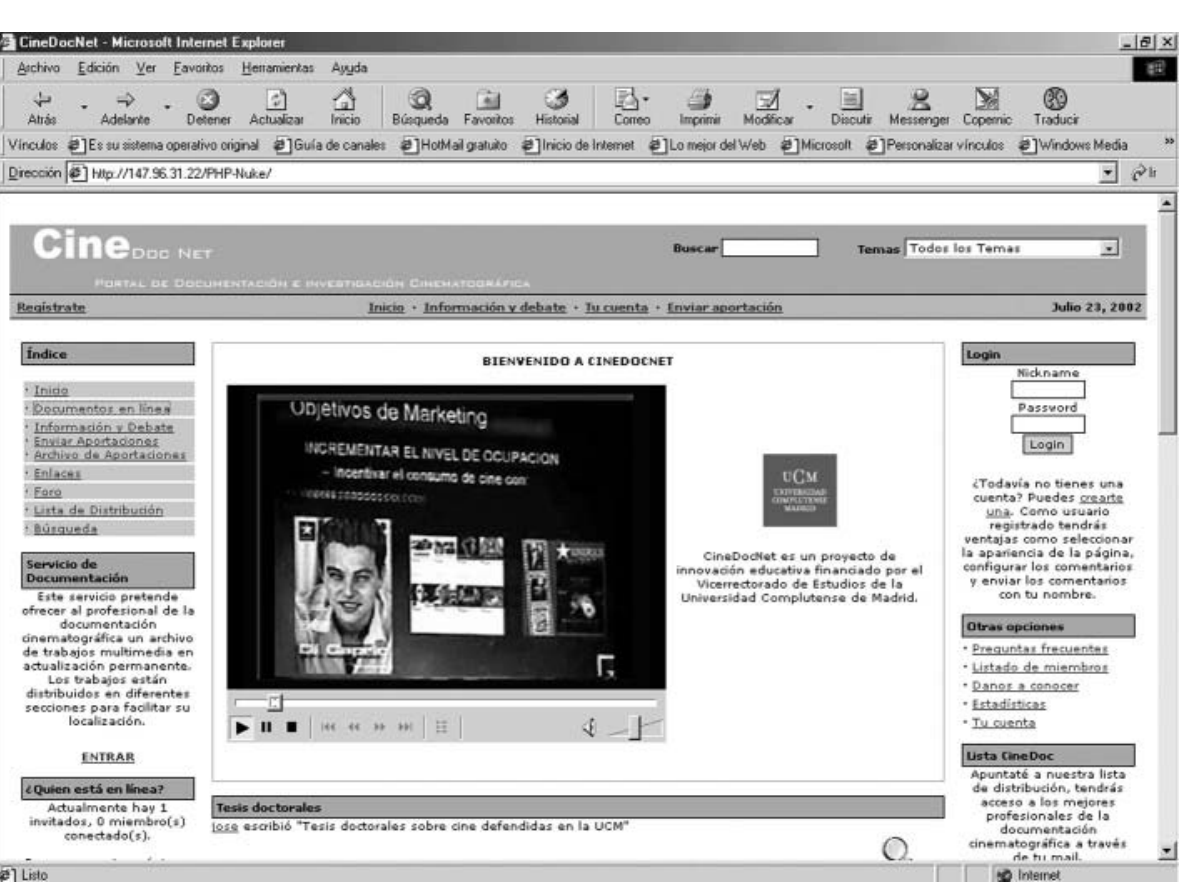

\section{Formación y consolidación de una CVU de documentación audiovisual}

El proceso para su creación debe dividirse en tres grandes etapas:

1. Fase estructural: se definen los objetivos fundamentales en previsión de los intereses de los futuros miembros y en función de los servicios que los creadores de la CVU quieren prestar, a modo de primera iniciativa dirigida al resto de miembros de la comunidad.

2. Incorporación de miembros: el segundo paso que se debe dar una vez establecidas las líneas 


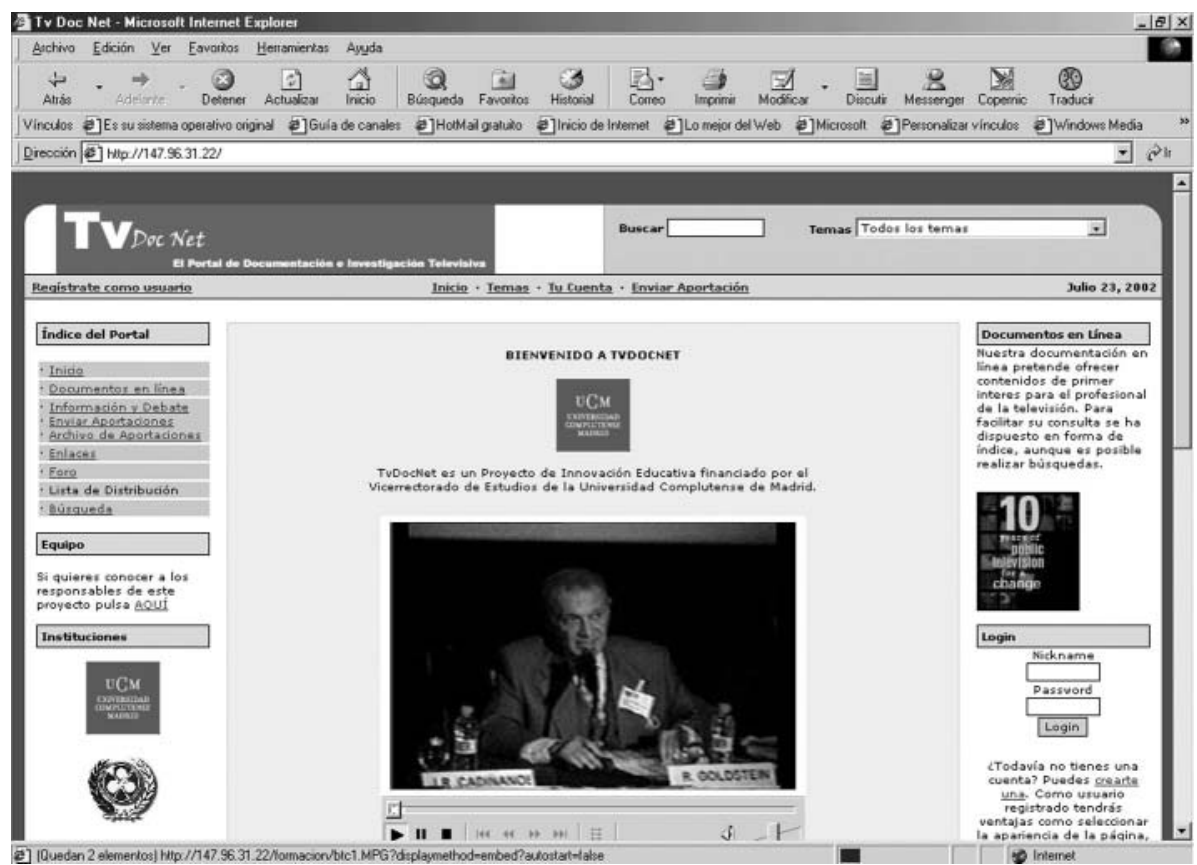

básicas que definirán a la CVU es la ampliación de miembros fuera del grupo inicial de trabajo.

3. Fase normativa: una vez producida la ampliación es necesaria una etapa de establecimiento de líneas de actuación para la consecución de los objetivos iniciales y la creación de otros nuevos dependiendo de las necesidades de los miembros incorporados a lo largo de la anterior segunda fase.

El cumplimiento de estas etapas se traduce en actuaciones concretas en lo referente a CineDocNet y TvDocNet, donde cada uno de las tres se suceden, repiten y superponen de forma mucho más dinámica de lo que se pueda expresar en lo sugerido en el gráfico 1. En la definición de objetivos, correspondiente a la fase estructural, uno de los problemas ha sido definir las líneas generales del proyecto proporcionando en todo momento un alto grado de escalabilidad a la iniciativa que nos permitiera adaptarnos a las variaciones, ampliaciones y especificaciones que se darían a lo largo de la consecución del proyecto. Ello nos ha llevado a situarnos en un nivel medio, la documentación audiovisual, lo suficientemente amplio como para incluir en ese ámbito temático dos categorías, como la documentación cinematográfica (CineDocNet) y la televisiva (TvDocNet), independientemente de la posterior inclusión en una definitiva CVU de documentación informativa, de otras temáticas relacionadas como son la docu- mentación periodística, la radiofónica y la publicitaria y de relaciones públicas.

De esta forma el esquema de la escalabilidad tanto del proyecto actual como de un proyecto futuro que englobaría todas las categorías mencionadas, presenta la estructura que se muestra en el citado gráfico 1 .

1. Factores de consolidación de la CVU de documentación audiovisual. En nuestra opinión, los puntos que garantizarán su afianzamiento son:

- Máxima claridad de objetivos, los cuales derivarán necesariamente en una mayor cohesión dentro de la CVU.

- Realización periódica de actividades de cohesión como congresos, teleseminarios, cursos en línea, etc., que aseguren no sólo el dinamismo del grupo, sino también la imbricación de los miembros de la CVU entre sí.

- Un alto grado de comunicación entre los miembros, posibilitado no sólo por los avances tecnológicos sino por la propia voluntad de los individuos de comunicarse. En este factor la implementación de herramientas específicas, de las cuales hablaremos más adelante, es de gran importancia.

2. Objetivos. Una vez estudiadas las sinergias necesarias para la implementación de una CVU de docu-

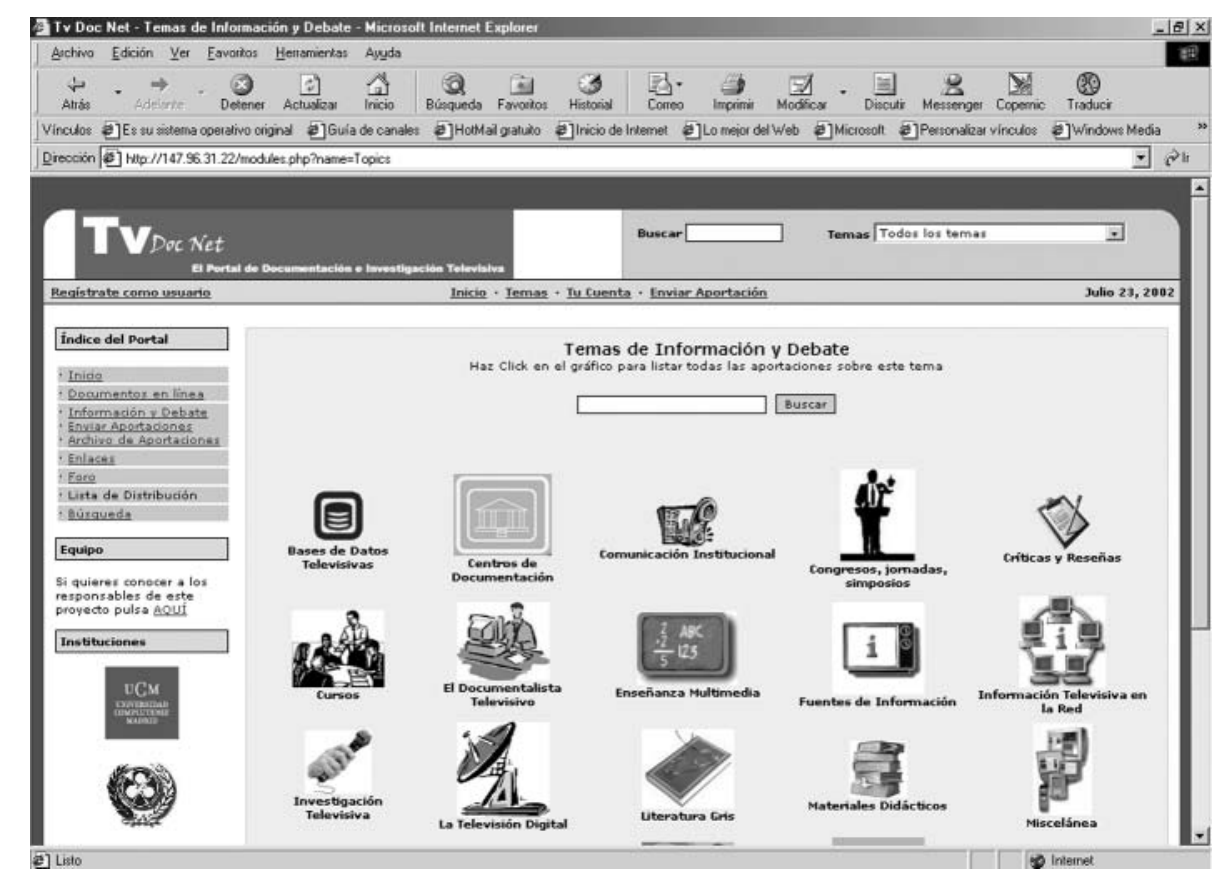


mentación audiovisual definimos las siguientes metas para nuestro proyecto:

- Servir de foro de debate y colaboración de los profesionales de la documentación y la investigación cinematográfica en torno a las ideas y planteamientos que existen sobre el tratamiento de este tipo de documentación.

- Ser un centro difusor de informaciones de carácter general y específico sobre documentación cinematográfica, ya sea en los ámbitos universitarios o en los profesionales.

- Transformarse en un lugar de discusión sobre trabajos de in-

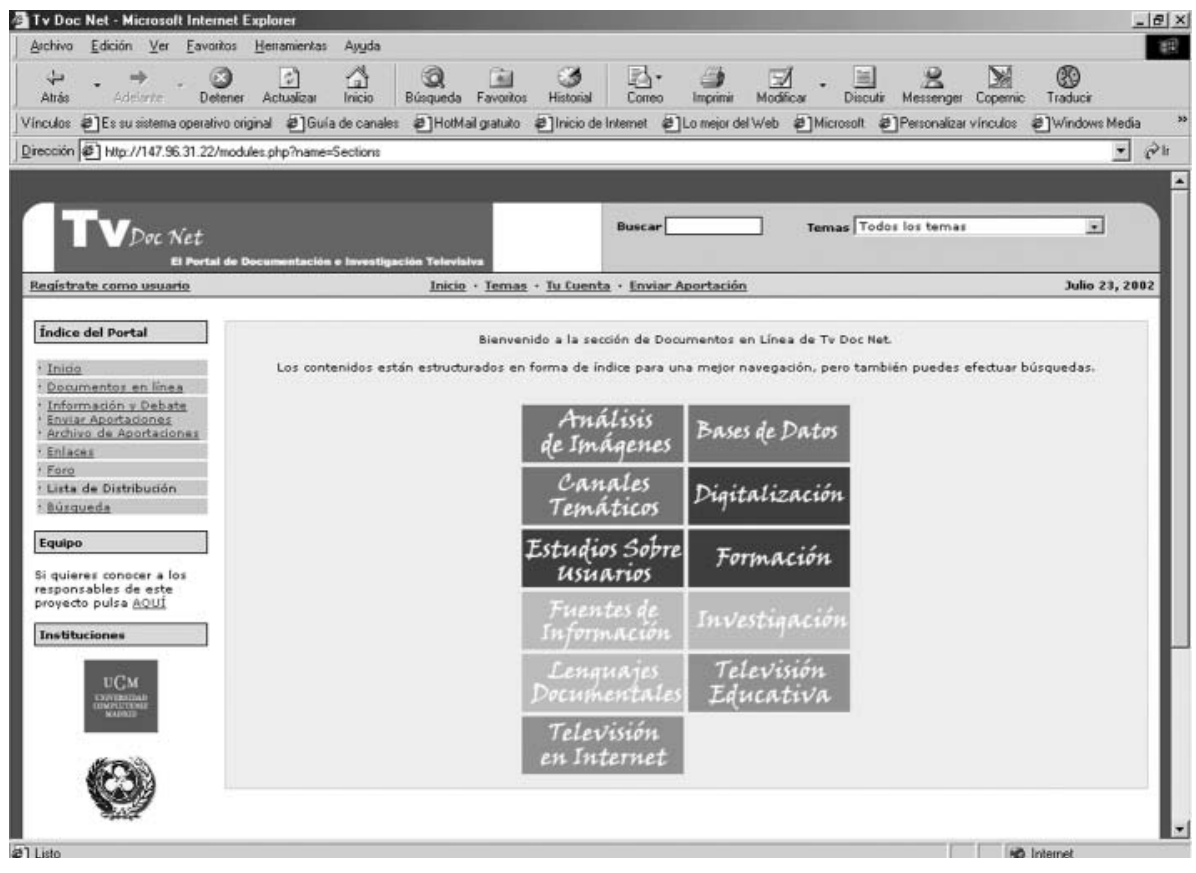
vestigación en documentación cinematográfica.

- Convertirse en un centro de intercambio de experiencias e innovaciones en el campo del tratamiento de este tipo de documentación, prestando una especial atención a la introducción de la informática e internet en su gestión.

- Ser, en suma, un espacio abierto de comunicación entre las personas interesadas en la mejora continua y progreso en las técnicas de tratamiento de documentación cinematográfica a todos los niveles, desde la universidad al ejercicio profesional ${ }^{5}$.

\section{Articulación de la CVU de documentación audiovisual: herramientas}

1. Weblog (o bitácora web). A modo de «diario de navegación», es una de las herramientas más novedosas que se han contemplado en la estructuración de funcionamiento de la CVU propuesta. En sí, un weblog contiene toda una filosofía que justifica sobrada-

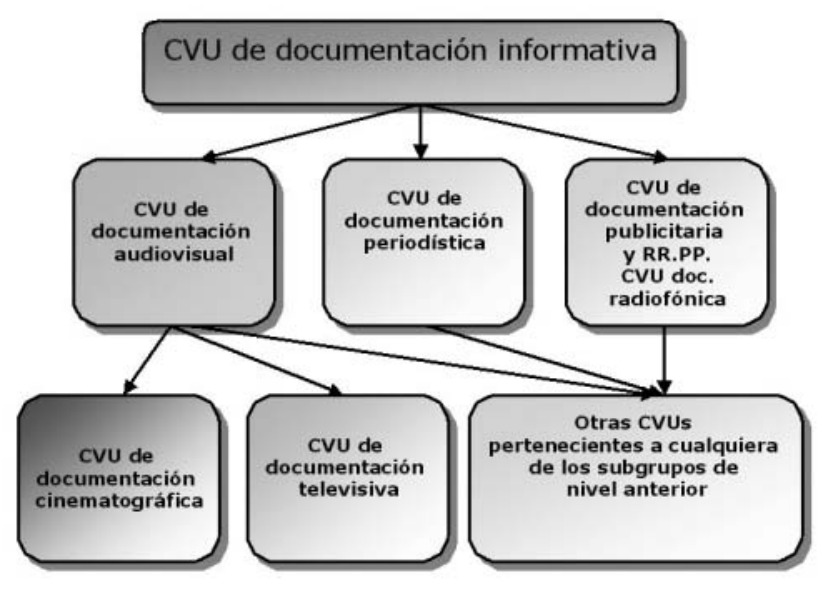

Gráfico 1

mente su utilidad. No nos vamos a extender aquí en esa vertiente, tratada de forma magistral por Javier Candeira en su artículo «La web como memoria organizada: el hipocampo colectivo de la web» ${ }^{3}$.

Fundamentalmente se trata de una forma de gestionar la información que la CVU recibe de cada uno de sus miembros. En el weblog de CineDocNet, al igual que en el de TvDocNet, cada uno de los miembros podrá hacer aportaciones de forma simple y rápida rellenando un sencillo formulario. Sus sugerencias serán publicadas en el portal contribuyendo a la generación de nueva información a partir de la ya existente. El almacenamiento de las aportaciones en una base de datos permitirá un fácil acceso y recuperación. De esta forma, el weblog se erige como una herramienta hecha por todos y para todos, prácticamente autogestionada y moderada tan sólo para evitar el ruido. En la tabla 1 se relacionan las temáticas sobre las que enviar aportaciones, modificables y ajustables en función de las necesidades que se vayan manifestando en CineDocNet.

La extensión de las contribuciones está limitada a 11.000 palabras, cantidad que resulta ampliamente suficiente. Además cada aportación puede tener un sentido diferente, ya sea de noticia, artículo, reseña, crítica, comentario, etc., y tan sólo aparece restringida por las temáticas a las que es necesario ceñirse, que como ya se ha mencionado pueden ser modificadas en función de las necesidades suscitadas.

Para el desarrollo de weblog, así como de los portales en general, hemos utilizado software $G N U$, fiable y gratuito, básicamente el servidor web Apache, el gestor de base de datos Mysql, el módulo PHP 4.0, además del sistema de creación de portales PHP-Nuke 


\begin{tabular}{|c|c|}
\hline $\begin{array}{l}\text { - Agencias de comunicación. } \\
\text { - Archivos filmicos y centros de } \\
\text { documentación. } \\
\text { - Bases de datos. } \\
\text { - Congresos, jornadas, etc. } \\
\text { - Cine digital. } \\
\text { - Consen internet. } \\
\text { - Críticas ciónematocupráficas. } \\
\text { - Criticas y reseñas. } \\
\text { - } \text { Dursos y seminarios. } \\
\text { - El director artístico. } \\
\text { - } \text { El director de fotografia. } \\
\text { - Distribución. } \\
\text { - Editonciales y librerías. }\end{array}$ & $\begin{array}{l}\text { - Estrenos. } \\
\text { - Exhibición. } \\
\text { Festivales. } \\
\text { - Filmotecas. } \\
\text { Formación. } \\
\text { - } \text { Investiganista. } \\
\text { audiovisuales. } \\
\text { - El montador. } \\
\text { - Patrimonio. } \\
\text { - Premios. } \\
\text { - Producción. } \\
\text { - Publiedad intelectual. } \\
\text { - Servicios de la industria. } \\
\text { - Tesis doctorales. }\end{array}$ \\
\hline
\end{tabular}

5.5, el cual hemos modificado para adaptarlo a nuestras necesidades.

2. Documentos en línea. Otro de los elementos imprescindibles en la articulación de la CVU son los documentos en línea. Este servicio pretende ser un espacio que recoja materiales seleccionados relacionados con la documentación cinematográfica o televisiva según se trate de CineDocNet o TvDocNet. En este caso, nuestro principal objetivo ha sido el de mostrar a la comunidad todas las posibilidades de la Red. Para ello hemos incluido gran cantidad de información, no sólo en formato texto, sino también en vídeo wmv (Windows media video), entre otros, para ser distribuido mediante streaming. Los materiales del servicio documental han sido clasificados en fichas a las cuales se accede en formato html y donde se puede encontrar información básica sobre el recurso al que dan acceso. Un ejemplo de las fichas indexadas - e incorporadas a base de datos para facilitar su localización, pudiendo ser recuperadas mediante un motor de búsqueda - podemos verlo en la tabla 2.

El recurso es almacenado en diferentes servidores en función de su formato. Para los documentos pdf el sistema es sencillo, ya que cada ficha está enlazada directamente al documento al que hace referencia. Para los archivos de vídeo se ha utilizado Kasenna, que nos permite su gestión y distribución por internet, localizada en los servidores de RedIris, que asimismo proporcionan el servicio de streaming. Los archivos en formato wmv cuentan con una doble indexación, por un lado la ficha de la base de datos Mysql que da acceso directo al recurso y por otro lado el asset (vídeo + metainformación) propiamente dicho al que también se puede acceder mediante consulta a la base de datos Kasenna.

Todos estos materiales constituyen una base documental or- ganizada y seleccionada de utilidad para los estudiantes y profesionales de la documentación cinematográfica y televisiva, además de mostrar que las posibilidades de internet van mucho más allá de la presentación de texto en diferentes formatos. Nuestro apartado de documentos en línea se estructura en las siguientes secciones:

- Cine en internet.

-Documentación y bases de datos.

-Formación.

-Fuentes de información.

-Investigación.

-Industria.

-Estudios monográficos.

En el caso de TvDocNet los documentos en línea se dividen de la siguiente forma:

- Bases de datos.

-Lenguajes documentales.

-Análisis de imagen.

-Investigación.

- Televisión educativa.

-Canales temáticos.

-Televisión en internet.

-Digitalización.

-Fuentes de información.

-Formación.

-Estudios de usuarios.

Además de la localización por secciones, existe otra forma de recuperar información mediante un motor de búsqueda documental. La intención es fomentar

\begin{tabular}{|l|l||}
\hline $\begin{array}{l}\text { Título: Comercialización en vídeo, DVD, } \\
\text { televisión e Internet }\end{array}$ & Tema: Marketing Cinematográfico \\
\hline Tipo de documento: Texto & $\begin{array}{l}\text { Feha de publicación del original: } \\
\text { Marzo 2001 }\end{array}$ \\
\hline Formato: PDF & $\begin{array}{l}\text { Lugar de trabajo del autor/s: TVE, } \\
\text { Telecinco, UAM, Federación para Ia } \\
\text { Protección de Propiedad Intelectual, } \\
\text { Warner Home Video, Conecta. }\end{array}$ \\
\hline Idioma: Castellano & $\begin{array}{l}\text { Autor/s: José Ramón Rubio, Yolanda } \\
\text { Marugan, Ignacio Redondo, Javier } \\
\text { Hernando Capón, Marisa Portugués, } \\
\text { Andrés González. }\end{array}$ \\
\hline
\end{tabular}

Resumen del contenido: El texto las intervenciones sobre comercialización del cine que tuvieron lugar el jueves 8 de marzo de 2001 durante las II Jornadas de Marketing Cinematográfico.

Fuente: II Jornadas de Marketing Cinematográfico organizadas por el Club de Dirigentes de Marketing de Madrid y patrocinadas por el Ministerio de Educación, Cultura y Deporte. Ed. Servicio de Documentación Multimedia UCM.

Palabras clave: cine, video, DVD, televisión, internet. 
iniciativas de digitalización de documentación multimedia por parte del resto de usuarios, para que los recursos de la CVU sean aportados por todos ellos, no sólo por medio de aportaciones en el weblog, sino a través de propuestas más complejas y elaboradas que la doten de una mayor riqueza de contenidos, haciéndola útil para los profesionales de la documentación audiovisual. Pensamos que de esta forma se posibilita una verdadera y eficaz interactividad.

3. Chat y videoconferencia. Tanto el weblog como el servicio de documentación son herramientas de tipo diacrónico pensadas para mantener el contacto entre los miembros de la CVU sin necesidad de la coincidencia de las coordenadas temporales. Ahora bien, para la implementación de los requisitos teóricos anteriormente mencionados es útil la comunicación sincrónica. Para ello contamos con dos servicios complementarios: la textoconferencia o chat, desarrollado gracias a la colaboración de RedIris que nos presta su sistema Volano, y la videoconferencia, donde la herramienta web Vrvs (Virtual Rooms Videoconference System) de la Universidad de Berkeley, de la cual RedIris actúa como reflector, constituye la solución perfecta para las necesidades de comunicación sincrónica de la CVU.

La utilización masiva de estas aplicaciones pertenece a la fase de consolidación, si bien pensamos que deberán estar disponibles desde el principio para mostrar las posibilidades de interrelación reales que ofrece el sistema, así como otros usos potenciales del mismo: formación virtual, congresos, jornadas, debates, mesas redondas, etc.

4. Foros de discusión-listas de distribución. Las listas de distribución han sido para la comunidad académica y científica la base en las que se asientan las CVUs. El papel desempeñado por RedIris (Jesús Sanz de las Heras) para la difusión y desarrollo de este medio de comunicación ha sido crucial para la formación de lo que se ha dado en llamar redes temáticas o CVUs. El funcionamiento de este tipo de herramientas es bastante sencillo, y tan sólo requiere que los usuarios se registren, siempre de forma gratuita, antes de poder formar parte de la lista de distribución.

Su dinámica de uso basada en el e-mail es enormemente fluida, y entre sus miembros se distribuyen diariamente todos aquellos mensajes que hayan sido enviados a la lista por cualquiera de los usuarios dados de alta. La ruptura de fronteras físicas que supone la utilización del correo electrónico permite la comunicación con personas de cualquier parte del mundo, con lo cual el grado de proyección de los mensajes es mucho mayor, permitiendo la comunicación entre científicos y profesionales independientemente del lugar geográfico en el que se encuentren.
El tipo de información que se distribuye a través de ellas es muy variado y los límites tan sólo están fijados por la temática general especificada en el momento de creación de las mismas. Así pues, podemos encontrar mensajes con bibliografías, celebración de congresos $^{4}$, desarrollo de proyectos, etc. Las intervenciones de los usuarios, a pesar de que en la mayoría de los casos están moderadas por los administradores responsables de la lista, gozan de absoluta libertad y cada uno puede reorientar las discusiones en función de sus intereses.

\section{«Las herramientas que articulan CineDocNet y TvDocNet son: servicio de documentación, we- blog, lista de distribución, foro, envío de aportaciones (modera- das) por parte de los usuarios y chats periódicos»}

Debido a este mecanismo de retroalimentación que constituye la base del funcionamiento de este tipo de servicios, su capacidad para generar nuevo conocimiento es ilimitado, y es aquí sin duda donde reside su mayor valor. Por esta razón es importante que, aparte de los sistemas de distribución, deban contar con un mecanismo de gestión y recuperación de la información que haga posible el almacenamiento en una base de datos de los mensajes enviados y su fácil recuperación retrospectiva. En el contexto de RedIris se ofrece esta solución, lo cual está permitiendo la creación automática de enormes archivos, clasificados en función de las temáticas que definen a cada una de las listas, que contienen gran cantidad de información útil para el desarrollo del conocimiento científico y del ejercicio profesional.

Otra de las ventajas de la utilización de listas de distribución es el alto grado de horizontalidad que existe en la relación entre sus usuarios. La falta de una jerarquía más allá de los administradores es total, lo cual permite una relación de igual a igual entre todos los miembros con el consiguiente aumento de la accesibilidad a cada uno de los participantes que esto supone. En nuestro caso, para la formación de una CVU de documentación audiovisual hemos optado por la creación de dos listas: CineDoc y TvDoc. La primera lleva funcionando desde 1999, mientras que la segunda es reciente y su existencia se debe a la necesidad que existe en una CVU de documentación audiovisual de hacer subdivisiones que se ajusten mejor a las necesidades de cada uno de los profesionales especializados dentro del campo de la documentación audiovisual.

http://www.rediris.es/list/info/cinedoc.es.html 
Si bien la presencia de una lista de distribución es crucial a la hora de crear una CVU, en nuestra opinión se hace necesario otro tipo de servicios que amplíen la capacidad de comunicación entre sus miembros, y permita nuevas formas de comunicación aprovechando al máximo las posibilidades que ofrece internet. Por esta razón, cuando pensamos en el diseño de la CVU concedimos a la lista de distribución un papel necesario pero no suficiente y decidimos complementarla con otras aplicaciones.

5. Foros vía web. Con el objetivo de facilitar el debate en la CVU hemos incluido una opción para la creación de foros vía web, gestionados también a través de una base de datos, que permite a los usuarios de la CVU acotar sus líneas de debate y crear por sí mismos debates a partir de las herramientas básicas que ofrece el portal. Los foros pueden ser creados y moderados por cualquier usuario permitiendo una mayor interacción entre ellos y el entorno que ha sido programado, que intenta ser lo más interactivo posible.

\section{«Estas comunidades virtuales se enmarcan, como primera ac- tuación, en el proyecto 'Red es- pañola e iberoamericana de do- cumentación multimedia', su- gerido desde el Departamento de biblioteconomía y documen- tación de la UCM»}

La ventaja que ofrecen con respecto a la listas es la mayor capacidad de acotación y jerarquización de la información. De esta forma, se constituye como un elemento complementario a la lista de distribución donde desarrollar discusiones surgidas en su entorno y que pueden seguir siendo mantenidas en la web evitando ruido a los usuarios de la lista no interesados en una discusión concreta.

6. Enlaces. Es otro de los puntos de información indispensbles de las CVUs motivo de este trabajo, ya que nos permiten trascender los límites de las mismas y referenciar los recursos de interés que se encuentran en internet y que pueden ser de enorme utilidad a los profesionales de la documentación informativa. Este apartado de enlaces también está gestionado mediante una base de datos y ha sido articulado en las siguientes secciones:

-Asociaciones y federaciones: asociaciones de actores, federaciones de productores, federaciones internacionales.

-Certámenes y festivales: tanto de España como del extranjero.

-Congresos, jornadas y seminarios.

-Ferias y exposiciones.

-Formación-enseñanza: bases de datos, bibliografía y fuentes de referencia, centros de formación.

-Industria: distribución, exhibición, guías de la industria audiovisual.

-Películas: carteleras, carteles de películas, cortometrajes.

-Investigación y tratamiento documental: ámbitos de investigación, archivos fotográficos, archivos, bibliotecas, centros de información y documentación.

-Portales y otros servicios en internet: chats, CVUs, listas de distribución.

-Profesionales: actores y actrices, animación, directores.

- Publicaciones y bases de datos: bases de datos (cd-rom, dvd y Red), cine en medios de comunicación.

- Tecnología documental: hardware y software, aplicaciones, producción y postproducción digital.

- Webs temáticas: bandas sonoras, canales temáticos sobre cine, cine en general.

La introducción de enlaces también puede ser llevada a cabo por cualquiera de los miembros de la CVU permitiendo una continua actualización de la sección, fundamental para este tipo de recursos.

\section{Próximos números monográficos}

\section{Noviembre 2002 Comercio electrónico y gestión de la información \\ Enero 2003 Perfil profesional y mercado de trabajo \\ Marzo 2003 Estándares documentales en internet: Z39.50, Dublin Core...}

Los interesados pueden remitir notas, artículos, propuestas, publicidad, comentarios, etc., sobre estos temas a:

epi@sarenet.es 
7. Groupware Bscw. Con la consolidación de la CVU motivo de estas páginas aumentará la necesidad de interacción entre sus miembros, siendo de gran utilidad para dicho requerimiento el uso de aplicaciones de trabajo colaborativo y más concretamente $B s c w$ (Basic support for co-operative work) ofrecida por RedIris. Dichas herramientas aparecen aquí incorporadas gracias al importante papel de esta red académica española en el desarrollo de nuestro proyecto. No es momento ahora de entrar en detalles acerca de su utilización, sobre la cual ya escribieron un interesante artículo Víctor Castelo y Pedro Miguel Ruiz Martínez publicado en el número 8 de Cuadernos de documentación multimedia. Tan sólo decir que $B s c w$ permite la formación de grupos de trabajo dentro de la CVU y facilita la colaboración mediante un entorno servido vía web preparado para administrar el flujo de trabajo de los miembros del equipo mediante aplicaciones diacrónicas.

Hasta ahora se venía utilizando el correo electrónico para el intercambio de información entre investigadores. Pero en los últimos años han surgido nuevas herramientas de trabajo cooperativo ${ }^{7}$ adaptadas a internet. La función principal de estos sistemas es crear una zona de trabajo virtual en red que permita a los miembros de un equipo de investigación operar de forma conjunta independientemente de su localización geográfica. Su funcionamiento se basa en la creación de una página web de acceso restringido que permite un alto grado de interactividad, pudiendo cada uno de los miembros del equipo articular completamente su actividad a través de la aplicación.

\section{«El proceso de formación de la CVU de documentación audio- visual se articula en tres fa- ses: de estructuración, de in- corporación de miembros, y normativa»}

La estructura de la zona de trabajo se establece a partir de carpetas donde se pueden ir depositando los documentos y las informaciones que se quieren transmitir al resto del equipo. De esta forma se constituye también en un depósito de materiales referentes al proyecto, los cuales a su vez pueden ser actualizados sin pérdida de información, ya que todas las acciones que se llevan a cabo en la zona quedan registradas y anotadas para que todos los miembros estén informados de las modificaciones realizadas.

Esta aplicación permite, entre otras cosas, la creación de un calendario y un plan de trabajo, la utilización de sistemas de reunión electrónica como son la videoconferencia ${ }^{8}$ y el chat, la coordinación y utiliza- ción del correo electrónico a partir de la propia zona de trabajo, el almacenamiento de conocimiento de forma que, en todo momento, se encuentra accesible a todos sus miembros, la escritura en grupo y la edición compartida de textos. Todas estas opciones permiten al equipo de investigación trabajar al mismo nivel que si se encontraran en la misma demarcación física, ya que el flujo de trabajo no cesa prácticamente nunca y además se mantiene siempre actualizado y ordenado.

\section{Conclusiones}

En este artículo se muestran las líneas generales en las que nos hemos basado para la creación de una CVU de documentación informativa de la cual ya es realidad una primera fase constituida por los portales CineDocNet y TvDocNet, enmarcados en un Proyecto de Innovación Educativa del Vicerrectorado de Estudios de la Universidad Complutense de Madrid, desarrollados en el ámbito del Servicio de Documentación Multimedia (Multidoc) de dicha universidad. La característica central de la CVU es su posibilidad de retroalimentación, lo que la convierte en un sistema de gestión y producción de nuevo conocimiento y de su difusión, fundamental para adaptarse a las necesidades sociales que operan en la nueva sociedad de la información.

\section{Notas}

1. http://www.well.com

2. Castells, Manuel. La era de la información: economía, sociedad y cultura. Madrid: Alianza Editorial, 1998.

3. Para poder pensar (monográfico). Revista de Occidente, 2001, marzo 2001.

http://jamillan.com/para_can.htm

4. Aparte de cada una de las listas, RedIris ofrece a la comunidad científica un canal de distribución de eventos llamado Diseven, a través del cual informa sobre la celebración de jornadas, mesas redondas, seminarios y en general todo tipo de eventos de similares características.

5. Este esquema está basado en el recogido para la lista Edumat por Juan D. Godino y Jesús Enfedaque en su artículo «Comunidad iberoamericana virtual de educación matemática».

http://www.rediris.es/cvu/publ/Civem.htm

6. http://www.ucm.es/info/multidoc/multidoc/revista/num8/castelo.html

7. Cscw (Computer support for colaborative work).

8. Castelo Gutiérrez, Víctor; Ruiz Martínez, Pedro Miguel. "Videoconferencia y Bscw: la base tecnológica para la formación en línea y el trabajo en grupo". En: Cuadernos de documentación multimedia, 1999, n. 8. Consultado en: 20-06-02.

http://www.ucm.es/info/multidoc/multidoc/revista/num8/castelo.html

Alfonso López Yepes; José Ramón Pérez Agüera, Dpto. de Biblioteconomía y Documentación, Facultad de Ciencias de la Información, Universidad Complutense de Madrid.

alopez@ccinf.ucm.es

thepolice@worldonline.es 